INPLASY

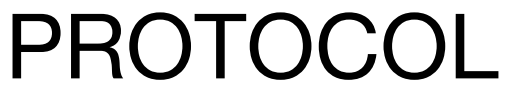

To cite: Wu et al. Tai Chi on bone mineral density of postmenopausal osteoporosis: a protocol for systematic review and meta-analysis. Inplasy protocol 202070104. doi:

10.37766/inplasy2020.7.0104

Received: 23 July 2020

Published: 23 July 2020

Corresponding author:

Yiru Wang

wangyiruen@163.com

Author Affiliation:

Longhua Hospital

Support: Shanghai Huangpu research.

Review Stage at time of this submission: The review has not yet started.

Conflicts of interest:

The authors declare that they have no competing interests.

\section{Tai Chi on bone mineral density of postmenopausal osteoporosis: a protocol for systematic review and meta-analysis}

Wu, HY1; Wang, YR²; Wen, GW3; Tang, ZY4; Yu, YQ5; Zhang, JR6; Liu, $\mathrm{P}^{7} ; \mathrm{Wu}, \mathrm{JH}^{8}$.

Review question / Objective: Does Tai Chi improve bone mineral density of postmenopausal osteoporosis?

Condition being studied: Osteoporosis is the most common bone disease. It is characterized by low bone mass, damage of bone microstructure, increase of bone fragility, and easy occurrence of fractures. As a therapeutic exercise, Tai Chi has been practiced for centuries as a martial art in China. At the same time, it has been drawn more and more attention. After introduced to Europe and America, the viewpoints of Tai Chi shifted and it is nowadays well-known as a kind of exercise or gymnastics.

Information sources: PubMed, Cochrane Library, Web of Science, China National Knowledge Infrastructure, Chinese Biological and Medical database and Wanfang Database.

INPLASY registration number: This protocol was registered with the International Platform of Registered Systematic Review and Meta-Analysis Protocols (INPLASY) on 23 July 2020 and was last updated on 23 July 2020 (registration number INPLASY202070104).

\section{INTRODUCTION}

Review question / Objective: Does Tai Chi improve bone mineral density of postmenopausal osteoporosis?
Condition being studied: Osteoporosis is the most common bone disease. It is characterized by low bone mass, damage of bone microstructure, increase of bone fragility, and easy occurrence of fractures. As a therapeutic exercise, Tai Chi has been practiced for centuries as a martial art in 
China. At the same time, it has been drawn more and more attention. After introduced to Europe and America, the viewpoints of Tai Chi shifted and it is nowadays wellknown as a kind of exercise or gymnastics.

\section{METHODS}

Participant or population: Postmenopausal osteoporosis.

Intervention: Tai Chi.

\section{Comparator: Without Tai Chi.}

Study designs to be included: Randomized controlled trial.

Eligibility criteria: All patients should be postmenopausal women and also meet the osteoporosis diagnostic criteria established by the Scientific Advisory Board of the European Society for Clinical and Economic Aspects of Osteoporosis (ESCEO) and the Committees of Scientific Advisors and National Societies of the International Osteoporosis Foundation (IOF).

Information sources: PubMed, Cochrane Library, Web of Science, China National Knowledge Infrastructure, Chinese Biological and Medical database and Wanfang Database.

Main outcome(s): BMD (g/cm2) of the lumbar spine, Ward's triangle, femoral neck, proximal femur, trochanter, or total hip.

Additional outcome(s): Pain score, alkaline phosphatase (ALP), osteocalcin (OC) and adverse effects.

Quality assessment / Risk of bias analysis: Cochrane Collaboration's tool will be used to evaluate the risk of bias by two independent reviewers (HYW and YRW). The assessment contains seven points: sequence generation, allocation concealment, blinding of participants and personnel, blinding of outcome assessors, incomplete outcome data, selective outcome reporting, and other bias. There are three levels of each bias according to the Cochrane Handbook for Systematic Reviews of Interventions (Version 5.3): low, unclear and high level. The disagreements cannot be resolved in this review will search for a third author (JHW) as required. Or else, we will ask the Cochrane Professional Group for a final decision.

Strategy of data synthesis: We will use Chisquared test to assess homogeneity of the included studies. If 12 statistic $>50 \%$, we will consider that there is a significant heterogeneity of the test and use a random effect model; If 12 statistic $<50 \%$, it means that there is no statistical heterogeneity or heterogeneity is small relatively, therefore we will use a fixed effect model.

Subgroup analysis: We will perform subgroup analysis to measure and cope with the heterogeneity due to the following reasons: (1) Clinical consideration: different age and race; different frequency, course and duration of Tai Chi (2) Methodology consideration: tests with unclear or high risks of bias.

Sensibility analysis: We will perform the sensitivity analysis to test if possible lowquality studies included. The detailed method is to remove each included article or some types of articles, then test the 12 value. This is the main method to assess the robustness and reliability of the synthesized meta-analysis results.

Language: English.

Country(ies) involved: China.

Keywords: Tai Chi; postmenopausal women; osteoporosis; review; metaanalysis.

Contributions of each author:

Author 1 - Haiyang Wu.

Author 2 - Yiru Wang.

Author 3 - Guowei Wen.

Author 4 - Zhenyin Tang.

Author 5 - Yiqun Yu.

Author 6 - Jiren Zhang.

Author 7 - Ping Liu.

Author 8 - Junhao Wu. 